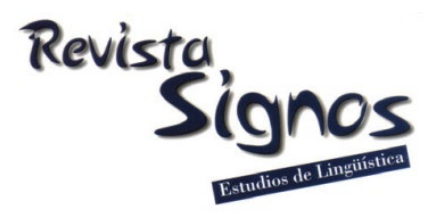

\title{
Los géneros en los textos de manuales escolares de ciencias naturales
}

\section{Genres in school textbooks of Natural Sciences}

\author{
Carina Alejandra \\ Rudolph \\ UNIVERSIDAD NACIONAL DE SAN JUAN \\ ARGENTINA \\ crudolph@ffha.unsj.edu.ar
}

\author{
Carla Inés Maturano \\ UNIVERSIDAD NACIONAL DE SAN JUAN \\ ARGENTINA \\ cmatur@ffha.unsj.edu.ar
}

María Amalia
Soliveres
Adiv NACIONAL DE SAN JUAN
ARGENTINA
soliver@ffha.unsj.edu.ar

Recibido: 22-IX-2018 / Aceptado: 22-V-2019

DOI: $10.4067 /$ S0718-09342020000200520

\section{Resumen}

Esta investigación se relaciona con los géneros de los textos escolares que se utilizan para aprender ciencias naturales, en especial física y química, en la escuela secundaria argentina. Considerando la necesidad de ocuparse de la lectura en el ámbito de las disciplinas, el docente de ciencias debería apropiarse de herramientas que le ayuden a deconstruir los textos y a proponer actividades relevantes para favorecer su comprensión. En este artículo aplicamos una tipología desarrollada en el marco de la Teoría de género de la Lingüística Sistémico-Funcional a una muestra de textos en español extraídos de manuales escolares de ciencias. El objetivo es brindar herramientas al docente de ciencias para categorizar los mismos como un primer paso para favorecer la construcción, tanto de conocimiento discursivo como de conocimiento disciplinar. Este artículo incluye una caracterización de los géneros informes y explicaciones, a partir de los aportes teóricos mencionados, cuyo análisis focaliza en el propósito, en las etapas de su estructura y en las realizaciones léxico-gramaticales distintivas de cada género. Se propone un análisis para textos escolares de física y de química en español, que ayudaría al docente disciplinar en los procesos de selección de textos y de diseño de actividades de lectura, como punto de partida necesario para su utilización en las clases.

Palabras Clave: Física y química, textos escolares, Lingüística Sistémico-Funcional, informes, explicaciones. 


\begin{abstract}
This research is related to the genres of school texts that are used to learn Natural Sciences, especially Physics and Chemistry, in secondary education in Argentina. Considering the need to deal with reading in the disciplines, we think that science teachers should have tools that help them deconstruct the texts and propose relevant activities to promote comprehension. In this article we apply a typology developed for science genres within the framework of Systemic Functional Linguistics Genre Theory to a sample of Spanish texts from school science textbooks. The objective is to provide tools to science teachers to categorize texts as a first step to favor the construction of both discursive and disciplinary knowledge. The article includes a characterization of two genres: reports and explanations from the aforementioned theoretical contributions focusing on their purpose, the stages of their schematic structure and their distinctive linguistic features. We propose an analysis of Physics and Chemistry school texts in Spanish, which could help the disciplinary teacher in the processes of selection of texts and design of reading activities accordingly, as a necessary starting point before they are used in class.
\end{abstract}

Key Words: Physics and Chemistry, school texts, Systemic Functional Linguistics, reports, explanations.

\title{
INTRODUCCIÓN
}

La enseñanza y el aprendizaje de las ciencias naturales involucran la comunicación a través de materiales escritos con características propias que expresan los contenidos disciplinares. La comprensión de los textos que se incluyen en dichos materiales ha sido objeto de numerosas investigaciones que reportan serios problemas en diversos contextos educativos (Lerner, Aisenberg \& Espinoza, 2010). En Argentina, algunos de estos problemas se manifiestan en los resultados de las Pruebas Aprender que muestran que el 36,3\% de los estudiantes logran resultados básicos o por debajo del nivel básico en ciencias naturales, lo que implica, entre otros factores, que tienen dificultades para interpretar información implícita presentada en textos breves de las disciplinas y para realizar inferencias a partir de estos (Ministerio de Educación y Deportes, MED, 2017).

En la escuela secundaria, los estudiantes deberían aprender los contenidos disciplinares, caracterizados por modos particulares de construir significados, para lo cual es necesario que desarrollen una alfabetización avanzada que les permita el acceso a textos que explican conceptos y relaciones como parte del contenido a aprender en cada disciplina (Schleppegrell \& Colombi, 2002; Moyano, 2013). El foco principal de estudio de esta investigación se relaciona con los géneros de los textos escolares que se utilizan para aprender ciencias naturales en la escuela secundaria argentina.

En numerosos contextos educativos, una de las principales fuentes de aprendizaje de contenidos científicos la constituyen los manuales escolares (Serrano, Moraga \& Lazo, 2011; Occelli \& Valeiras, 2013; Taibu, Rudge \& Schuster, 2015; Maturano \& 
Mazzitelli, 2018). Los textos de los manuales, particularmente los de física y de química, incluyen un complejo conjunto de relaciones entre los componentes verbales y visuales ya que en los mismos interactúan diversos sistemas semióticos: (1) un sistema verbal, que constituye la expresión de significados basados en lo lingüístico, exclusivamente; (2) un sistema gráfico que permite la presentación de datos en formatos tales como fotografías, diagramas, tablas, entre otros; (3) un sistema matemático conformado por grupos de grafías, signos o representaciones que permiten que el significado sea codificado simbólicamente de forma sintética; y (4) un sistema tipográfico constituido por la forma, tamaño y color de las letras (Parodi, 2010). La multimodalidad, compartida en ciertos aspectos con el texto de matemática, se representa a través de tres elementos: a) la disposición espacial de los símbolos, b) la representación del problema combinando simbolismo y escritura, y c) las microtrancisiones intersemióticas (Manghi, 2013). Esta compleja interacción implica para el estudiante enfrentar dificultades diferentes de las que se le presentan cuando lee en otro contexto y poner en práctica procesos inferenciales que demandan una mediación del docente (Maturano, Rudolph \& Soliveres, 2016). Así, los modos particulares de lectura propios de las disciplinas científicas deberían ser guiados por el docente de ciencias para acercar a los estudiantes a las características distintivas de los textos disciplinares (género, léxico específico, propósito de lectura, entre otros), mientras trabaja los contenidos de las asignaturas. Esto implicaría que el profesor tomara conciencia de su rol mediador para que los estudiantes alcancen la alfabetización y puedan aprender a partir de la lectura de textos multimodales (Manghi, Torres, Urrutia, Martínez, Vega \& Morales, 2016). Los autores mencionados sostienen que los materiales utilizados para la enseñanza constituyen un desafío para su interpretación por lo que requieren de un docente mediador con experiencia semiótica que intervenga en el proceso pedagógico para favorecer el aprendizaje.

Sin embargo, al analizar actividades propuestas por docentes disciplinares en torno a los textos escolares de ciencias, se encuentra que la mayoría de las tareas: (a) se encuadra en la etapa de poslectura, (b) se centra en la reproducción de información textual y (c) tiene como propósito evaluar la comprensión del texto o el aprendizaje del contenido disciplinar por parte del estudiante, sin una mediación del docente que favorezca estos procesos durante la lectura (Soliveres, Maturano \& Quiroga, 2015).

Por otra parte, Ibáñez, Moncada, Cornejo y Arriaza (2017) señalan que pocas investigaciones han abordado las implicancias que las características lingüísticas del texto escolar podrían tener en los procesos de enseñanza y aprendizaje. Al mismo tiempo, no hemos registrado estudios que brinden a los docentes disciplinares herramientas con fines didácticos para su uso en el aula para abordar textos escolares de física y de química escritos en español. El problema planteado requiere que el docente de ciencias se apropie de herramientas que le ayuden a analizar los textos escolares y a proponer actividades relevantes para favorecer su comprensión. Este 
estudio contribuiría en ese sentido, presentando un análisis sencillo que pueda permitirle al docente disciplinar la deconstrucción de dichos textos como paso previo necesario para su utilización en las clases.

Para tal fin, consideramos que la Lingüística Sistémico Funcional (LSF) proporciona un marco descriptivo e interpretativo que permite el análisis de productos auténticos de la interacción social (textos), a los que considera en relación con el contexto social y cultural en el cual se negocian. Dentro de este marco, en Latinoamérica, se han realizado investigaciones que han analizado, con distintos objetivos, textos de manuales escolares en ciencias sociales y naturales (Moss, Natale \& Oteiza, 2009; Moyano, 2013; Manghi et al., 2016; Ibáñez et al., 2017; Ibáñez, Moncada \& Arriaza, 2018). No obstante, resulta novedosa la aplicación que se realiza en este artículo de una tipología formulada para textos en inglés por Martin y Rose (2008) en el marco de la Teoría de género de la LSF a una muestra de textos en español extraídos de manuales escolares de ciencias permite categorizar los mismos desde un enfoque basado en los géneros de las disciplinas como un primer paso para favorecer la construcción, tanto de conocimiento discursivo como de conocimiento disciplinar.

En este artículo presentamos una caracterización de los géneros informes y explicaciones referidos específicamente a contenidos de física y de química a partir de los aportes teóricos de la LSF. Luego, incluimos un análisis estructural y lingüístico de tres informes y cuatro explicaciones como modo de acercamiento a los géneros de forma sencilla buscando su utilidad para el docente de ciencias. Posteriormente, concluimos el artículo con una descripción de los principales aportes de la propuesta y los desafíos que se abren a partir de esta.

\section{Marco teórico}

$\mathrm{El}$ análisis de los textos de manuales escolares requiere un posicionamiento teórico desde la Lingüística, en este caso, la Lingüística Sistémica Funcional. De acuerdo con Halliday y Martin (2005), la LSF en contraste con la gramática tradicional tiene un cierto número de rasgos que la convierten en una herramienta efectiva para investigar el discurso científico. En primer lugar, la LSF se orienta a la descripción del lenguaje como un recurso para construir significados, más que como un sistema de reglas. En segundo lugar, considera al texto, más que a las oraciones, como la unidad básica de negociación de significados. A su vez, enfatiza las relaciones entre los textos y los contextos sociales en que se producen. Estos rasgos se ven reflejados en la Teoría de género de la LSF que considera los géneros como procesos sociales, orientados a un propósito y planteados en etapas (Martin \& Rose, 2008; Rose \& Martin, 2012). En los últimos treinta años, investigadores pertenecientes a la denominada Escuela de Sydney (Veel, 2005; Halliday \& Martin, 2005; Wignell, Martin \& Eggins, 2005), han formulado 
generalizaciones sobre los géneros de la ciencia y han identificado cuatro grandes familias de géneros, cada una de las cuales intenta caracterizar y explicar acerca de fenómenos y procesos del mundo natural. Así, Martin y Rose (2008) distinguen entre informes, que describen el mundo natural; explicaciones, que se centran en cómo y/o por qué suceden los procesos naturales; procedimientos, que instruyen sobre cómo realizar una secuencia de actividades; y recuentos de procedimientos, que interpretan y recuentan lo observado o realizado. Estos autores señalan que entre los géneros más frecuentes utilizados en ciencias en la escuela secundaria se encuentran los informes y las explicaciones, los cuales juegan un rol complementario entre sí al explorar un tema. A continuación, caracterizamos estos dos géneros según el propósito, la estructura esquemática y los rasgos lingüísticos prototípicos rescatando los aspectos más sustanciales propuestos por Christie y Martin (2000), Veel (2000), Halliday y Martin (2005), Schleppegrell (2008) y Martin y Rose (2008).

Los informes caracterizan fenómenos, ya sea describiéndolos, clasificándolos o indicando su composición. Se pueden identificar tres tipos de informes:

- informe descriptivo: describe un fenómeno (entidad o actividad) centrándose en sus rasgos o características. La estructura esquemática del informe descriptivo se determina a partir de la identificación del fenómeno (que en ocasiones incluye la definición), seguida por la etapa de descripción que presenta los rasgos o características del mismo. Las realizaciones léxicogramaticales distintivas de este género son: grupos nominales específicos del género (fenómeno y sustantivos relacionados), grupos verbales en tiempo presente del indicativo que realizan la función de procesos relacionales (especialmente verbos ser y/o estar), grupos adjetivos (que caracterizan el fenómeno) y conectores lógicos (especialmente de adición).

- informe clasificatorio: clasifica miembros de una clase general en relación con un criterio o conjunto de criterios. La estructura esquemática del informe clasificatorio generalmente comienza enunciando una clase general seguida por las subclases, cuyo criterio de clasificación puede estar explícito o debe inferirse en la lectura. Sus rasgos lingüísticos característicos son: grupos nominales específicos del género (clase general y subclases), grupos verbales en tiempo presente del indicativo que realizan la función de procesos relacionales (especialmente verbos que hacen referencia a clasificación y verbos como ser y/o estar), frases preposicionales (que introducen el criterio de clasificación).

- informe composicional: se centra en los componentes de una entidad (las partes de un todo), los cuales pueden definirse o describirse. La estructura esquemática del informe composicional se determina a partir de la identificación de la entidad, seguida por los componentes de la misma. Este género se realiza mediante: grupos nominales específicos del género (entidad y sus componentes) y grupos verbales en tiempo presente del indicativo que 
realizan la función de procesos relacionales (especialmente verbos que hacen referencia a composición y verbos como ser y/o estar).

Las explicaciones se centran en actividades y explican cómo y/o por qué suceden los procesos. Este tipo de género involucra secuencias de causas y efectos. Una diferencia fundamental entre informes y explicaciones es el rol del tiempo en su estructura. Mientras que los informes describen, clasifican o indican los componentes de fenómenos sin que el tiempo sea un factor determinante, las explicaciones vinculan entre sí actividades, ya sea que sucedan al mismo tiempo o que unas antecedan a otras. Se pueden identificar distintos tipos de explicaciones entre las que se destacan:

- explicación secuencial: se focaliza en una secuencia simple de eventos entre los cuales se da una relación causal obligatoria. La explicación secuencial generalmente consta de una estructura esquemática en la cual se identifica el fenómeno seguido por la etapa de explicación donde cada evento o fase es antecedente del que le sigue. Sus rasgos lingüísticos son: grupos nominales específicos del género (especialmente procesos nominalizados), grupos verbales en tiempo presente del indicativo que realizan la función de procesos materiales (especialmente verbos de acción), frases preposicionales (circunstancias de lugar) y conectores lógicos (especialmente temporales).

- explicación factorial: explica un evento en base a múltiples factores contribuyentes. Las relaciones entre los eventos son causales o temporales. La estructura esquemática de la explicación factorial comienza con la identificación del fenómeno seguida por la etapa de explicación de los factores o causas contribuyentes. Cada factor constituye una fase de la explicación. Las realizaciones léxico-gramaticales son: grupos nominales específicos del género (fenómeno y factores), grupos verbales en tiempo presente del indicativo que realizan la función de procesos relacionales y conectores lógicos (especialmente causales).

- explicación de consecuencia: se centra en un evento simple que puede tener dos o más consecuencias en el mundo natural. En las explicaciones de consecuencia la estructura esquemática se determina con la identificación del fenómeno, el cual es la causa de la/las consecuencia/s que se explicitan. Este género se realiza mediante: grupos nominales específicos del género (evento y consecuencias generalmente nominalizadas), grupos verbales en tiempo presente del indicativo que realizan la función de procesos materiales (especialmente verbos de acción que refieren a consecuencias) y conectores lógicos (especialmente que expresan causa/consecuencia).

- explicación condicional: explica fenómenos cuyos efectos pueden ser diferentes dependiendo de condiciones variables. Las condiciones en su totalidad especifican la generalización del fenómeno. La estructura esquemática comienza 
identificando el evento seguido por las condiciones que determinan los efectos posibles. Sus rasgos lingüísticos son: grupos nominales específicos del género (procesos nominalizados), grupos verbales en tiempo presente del indicativo que realizan la función de procesos materiales y cláusulas condicionales.

Una vez caracterizados los informes y las explicaciones, como géneros frecuentes en los textos escolares de ciencias naturales, a continuación, presentamos en el Marco metodológico el proceso de selección de los manuales de ciencias naturales que constituyen la muestra y de los textos que se consideraron prototípicos de cada uno de los géneros incluidos en este artículo. Posteriormente, describimos las realizaciones léxico-gramaticales que se usaron en el análisis de los textos.

\section{Marco metodológico}

El objeto de estudio de esta investigación son textos de manuales escolares utilizados para la enseñanza y el aprendizaje de las ciencias naturales, específicamente de física y de química, en la Educación Secundaria Básica de Argentina. Para seleccionar los manuales escolares tuvimos en cuenta que fueran editados recientemente y que fueran utilizados por docentes del área en sus clases. Se consideran manuales recientes aquellos que han sido editados con posterioridad a la última reforma educativa en el marco de la ley vigente en Argentina (Ley Nacional de Educación o Ley 26.206 del año 2006) que divide la Educación Secundaria en dos ciclos. En todos los casos, los libros de texto seleccionados para su estudio pertenecen al Ciclo Básico, de carácter común a todas las orientaciones, y se utilizan generalmente en primero, segundo y/o tercer año de la Educación Secundaria, dependiendo de la jurisdicción. Seleccionamos los que han sido propuestos por las editoriales más recomendadas por docentes argentinos, según lo relevado en una investigación previa en la que participaron profesores de nivel secundario de diferentes provincias de Argentina (Maturano, 2018; Maturano \& Mazzitelli, 2018).

En virtud de estas consideraciones, elegimos una muestra de libros de texto sobre la cual se recolectaron los datos, constituida por cuatro manuales escolares (Carmona de Rey, Pusterla \& Viscarra, 2010; Bazo, Abellán, Caro \& Sellés, 2012; Taddei, Sobico \& Righini, 2015; Balbiano, Deprati, Díaz, Franco, Iglesias \& Molinari Leto, 2016). Posteriormente, analizamos textos incluidos en los mismos con el objetivo de seleccionar informes y explicaciones breves (tal como lo proponen Martin \& Rose, 2008) con un marcado predominio de los rasgos lingüísticos de cada género, en concordancia con las relaciones propias del contenido científico involucrado.

Para la asignación del género a los textos usamos, en primera instancia, una técnica de consenso entre tres investigadores. Una vez decididos los rasgos prototípicos a tener en cuenta para asignar el género, mediante un proceso que considera lineamientos de la Teoría de género de la LSF, cada investigador con formación específica en el área de Lingǘstica y/o de investigación en educación en ciencias, 
analizó si los textos informaban acerca de un fenómeno o lo explicaban. Esta decisión se sustentó en la identificación de los rasgos lingüísticos prototípicos de los informes y explicaciones propuestos en la tipología de Martin y Rose (2008). Luego, en sesiones de debate, el acuerdo entre los investigadores nos permitió estimar una confiabilidad satisfactoria del juicio de expertos en la asignación del género. En una segunda instancia, en diversos talleres de perfeccionamiento con docentes disciplinares examinamos las características de los textos ya analizados por los expertos e identificamos aquellos que presentan los rasgos distintivos de cada género con mayor claridad y se adecuan para el trabajo en el aula en el Ciclo Básico de la Educación Secundaria (Maturano, Soliveres, Guirado \& Quiroga, 2014; Guirado, Soliveres \& Maturano, 2015; Soliveres et al., 2015). Finalmente, seleccionamos los siete textos que se presentan en este trabajo como representativos de los géneros informes (descriptivo, clasificatorio y composicional) y explicaciones (secuencial, factorial, de consecuencia y condicional). Así, el proceso llevado a cabo complementa categorías teóricas predefinidas y rasgos lingüísticos particulares que fueron emergiendo a partir del análisis de los ejemplares genéricos.

La propuesta de análisis de los textos surge, de este modo, tanto del trabajo conjunto de investigadores con formación en Lingüística y en las disciplinas en cuestión (física y química) como de docentes de Educación Secundaria, e intenta proporcionar herramientas al docente de ciencias que le permitan aprovechar los recursos de la Lingüística para reflexionar sobre el género de los textos y así poder planificar actividades que se adapten a la organización de la información tal como se presenta en los textos de los manuales escolares. Las categorías no contemplan un análisis lingüístico exhaustivo en términos de todos los aspectos que podrían incluirse y fueron abordadas sin inconveniente por docentes de ciencias en diferentes talleres de perfeccionamiento. Esta opción, que deja de lado el examen detallado de otros rasgos lingüísticos de los textos, se fundamenta en que, de hacerlo, la complejidad de la herramienta podría llevar a que el docente de ciencias desestime la propuesta de trabajo, mientras que haciéndolo de modo básico, se cumplirían los objetivos trazados en cuanto a la necesidad de poner énfasis especial en aquellos recursos del texto que sirvan de disparadores para diseñar actividades acordes al género que favorezcan la comprensión del texto $y$, en consecuencia, el aprendizaje de los contenidos disciplinares.

En la investigación realizada, las categorías de análisis se desprenden de los rasgos distintivos detallados en el marco teórico para cada uno de los géneros seleccionados. Estas categorías se refieren a: (a) el género del texto; (b) las etapas que conforman la estructura esquemática del texto y (c) los rasgos lingüísticos propios del género. En relación con la categoría de análisis (a), nos preguntamos si se describe o se explica un fenómeno; una vez determinado el género, en relación con la categoría (b), analizamos el despliegue de la información tratando de identificar las etapas descriptas para cada 
género; finalmente, respecto de la categoría de análisis (c), identificamos las realizaciones léxico-gramaticales prototípicas del género propuestas por la LSF.

A continuación, describimos, siguiendo a Halliday y Matthiessen (2004), Ghio y Fernández (2008) y Derewianka (2013), las realizaciones lingüísticas consideradas en el análisis de los textos de la muestra. Clasificamos los grupos de palabras de acuerdo con el tipo de palabra que se identifica como componente central y que necesariamente tiene que estar presente para formar el grupo, distinguiendo entre:

- grupo nominal: es la expansión de un sustantivo, el cual tiene la función de núcleo en el grupo y puede estar precedido o seguido por una serie de palabras que lo caracterizan; por ejemplo, "las fuentes de energía". El grupo nominal cumple el rol de participante en la cláusula.

- grupo verbal: es la expansión de un verbo y puede estar compuesto por una o varias palabras pertenecientes a la clase gramatical verbo; por ejemplo, "se clasifican". El grupo verbal cumple el rol de proceso en la cláusula.

- grupo adjetivo: tiene un adjetivo como núcleo, el cual puede o no estar acompañado por elementos que lo modifiquen; por ejemplo, el adjetivo "impermeables" o el grupo "fáciles de moldear". En los informes descriptivos, el grupo adjetivo suele cumplir el rol de complemento.

Otros términos que consideramos necesario identificar a los fines de este trabajo para facilitar al docente de ciencias el uso de esta propuesta, son:

- procesos relacionales: son procesos de ser, más específicamente de atribuir o identificar. Relacionan fragmentos de la experiencia; por ejemplo, "son", "está formada".

- procesos materiales: son procesos de hacer y acontecer e implican una acción o un evento. Conceptualizan la experiencia externa al individuo; por ejemplo, "transforman", "impactan".

- nominalización: es el proceso por el cual un evento no se realiza como verbo sino como sustantivo; por ejemplo, “corrosión” en vez de corroer.

- conectores lógicos: son palabras o grupos de palabras que muestran las relaciones lógicas o espacio-temporales que se establecen entre las ideas desarrolladas; por ejemplo, "sucesivamente", "luego".

- cláusula condicional: indica las condiciones bajo las cuales puede tener lugar un suceso, suele estar introducida por la conjunción si; por ejemplo, "Si el volumen del gas permanece constante...".

- frase preposicional: consiste en una preposición seguida por un grupo nominal; por ejemplo, "en los reactores nucleares". La frase preposicional puede cumplir el rol de circunstancia en la cláusula. 
La forma de trabajo propuesta llevaría al docente disciplinar a considerar en primer lugar los rasgos singulares de cada género para, posteriormente, captar la complejidad que caracteriza a los entramados típicos de los textos científicos. En este artículo se examinan los siete textos prototípicos a la luz de los siguientes cuestionamientos: ¿de qué trata el texto?, ¿cuál es el género del texto?, ¿cómo está organizada la información en etapas? y ¿qué realizaciones léxico-gramaticales confirman el género?

\section{Resultados y discusión}

En esta sección analizamos los géneros seleccionados, comenzando con los informes y siguiendo con las explicaciones en el orden en que se presentaron en el marco teórico. Esto es, en primer lugar, examinamos los tres textos que informan sobre fenómenos y, posteriormente, los cuatro textos que explican fenómenos. En cada caso se trata de textos transcriptos en este trabajo de forma literal, respetando el sistema tipográfico del manual escolar original. Las Tablas 1 a 7 incluyen los textos considerados como prototípicos (columna derecha) y el análisis realizado indicando el género, las etapas de la estructura esquemática de cada género (columna izquierda) y los rasgos lingüísticos de los ejemplares genéricos (fila inferior).

Asimismo, incluimos luego del análisis de cada texto, representaciones gráficas para cada género, las cuales sirven no sólo para mostrar la organización y la relación entre las ideas en forma general, sino también para confirmar el género del texto durante el proceso de análisis (Figuras 1, 3, 5, 7, 9, 11 y 13). Además, presentamos las propuestas para los textos de esta muestra que hacen explícita la organización del contenido disciplinar (Figuras 2, 4, 6, 8, 10, 12 y 14).

Tabla 1. Ejemplo de informe descriptivo.

\begin{tabular}{|l|l|}
\hline $\begin{array}{l}\text { Estructura esquemática: } \\
\text { etapas }\end{array}$ & \multicolumn{1}{c|}{ Texto 1} \\
\hline $\begin{array}{l}\text { Identificación de la } \\
\text { entidad }\end{array}$ & $\begin{array}{l}\text { Los plásticos } \\
\text { La palabra plástico hace referencia a un grupo de materiales sintéticos } \\
\text { capaces de entrar en un estado plástico; es decir, que pueden ser modelados } \\
\text { en estado líquido y que, en estado sólido, conservan la forma dada. Los más } \\
\text { conocidos son el nailon, el poliestireno, el policloruro de vinilo (PVC) y el } \\
\text { polietileno. Todos ellos son materiales sintéticos, es decir, transformados y } \\
\text { creados por el hombre. Los materiales plásticos son fáciles de moldear, } \\
\text { impermeables, buenos aislantes acústicos y mejores aislantes eléctricos; } \\
\text { además, son resistentes a la corrosión, entre otras propiedades (Bazo et al., } \\
\text { 2012, p. 20). }\end{array}$ \\
\hline $\begin{array}{l}\text { Realizaciones léxico-gramaticales específicas del género } \\
\text { Grupos nominales específicos: los plásticos (entidad), un grupo de materiales sintéticos, el nailon, el } \\
\text { poliestireno, el policloruro de vinilo, el polietileno, los materiales plásticos }\end{array}$ \\
$\begin{array}{l}\text { Grupos verbales en presente del indicativo que realizan la función de procesos relacionales: son } \\
\text { Grupos adjetivos que caracterizan la entidad: fáciles de moldear, impermeables, resistentes a la corrosión } \\
\text { Conectores lógicos de adición: y, además }\end{array}$ \\
\hline
\end{tabular}


entidad

definición

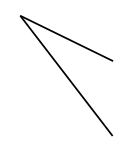

rasgo o característica 1

rasgo o característica 2

Figura 1. Propuesta propia de representación gráfica del informe descriptivo.

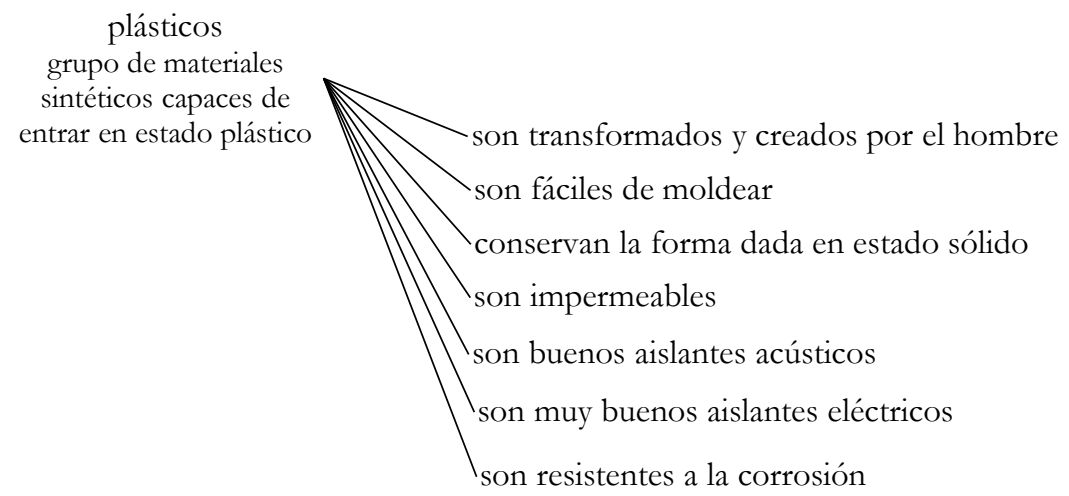

Figura 2. Propuesta de representación gráfica del texto 1.

Tabla 2. Ejemplo de informe clasificatorio.

\begin{tabular}{|c|c|}
\hline $\begin{array}{l}\text { Estructura } \\
\text { esquemática: } \\
\text { etapas }\end{array}$ & Texto 2 \\
\hline $\begin{array}{l}\text { Identificación de } \\
\text { la clase general } \\
\text { Criterio de } \\
\text { clasificación }\end{array}$ & $\begin{array}{l}\text { Fuentes de energía } \\
\text { Todos los cuerpos que suministran energía para ser utilizada en diferentes procesos } \\
\text { son denominados fuentes de energía. } \\
\text { De acuerdo con el desperdicio de esta energía y la imposibilidad de poder volver a } \\
\text { recuperarla, las fuentes de energía se clasifican en dos grandes grupos. } \\
\text { No renovables } \\
\text { Las fuentes no renovables son aquellas que existen en una cantidad limitada y que } \\
\text { con su uso terminan por agotarse, como los combustibles fósiles, el carbón, el gas, } \\
\text { y los combustibles nucleares, como el uranio. } \\
\text { Renovables } \\
\text { Las fuentes renovables son aquellas que no se agotan nunca, como el sol, el viento, } \\
\text { el agua, los géiseres. Son estas las fuentes cuya explotación conviene desarrollar, } \\
\text { porque prometen soluciones más duraderas, aunque no suficientes y, además, } \\
\text { presentan la ventaja de ser menos contaminantes (Carmona de Rey et al., 2010, p. } \\
\text { 53). }\end{array}$ \\
\hline \multicolumn{2}{|c|}{$\begin{array}{l}\text { Realizaciones léxico-gramaticales específicas del género } \\
\text { Grupos nominales específicos: fuentes de energía (clase general), las fuentes no renovables, las fuentes } \\
\text { renovables (subclases) } \\
\text { Grupos nominales específicos que se relacionan con las subclases: dos grandes grupos, los combustibles } \\
\text { fósiles, el carbón, el gas, los combustibles nucleares, el uranio, el sol, el viento, el agua, los géiseres } \\
\text { Grupos verbales en presente del indicativo que realizan la función de procesos relacionales: se clasifican, } \\
\text { son } \\
\text { Frases preposicionales: de acuerdo con (criterio de clasificación) }\end{array}$} \\
\hline
\end{tabular}


Figura 3. Propuesta de representación gráfica del informe clasificatorio, adaptado de Martin y Rose (2008: 146).

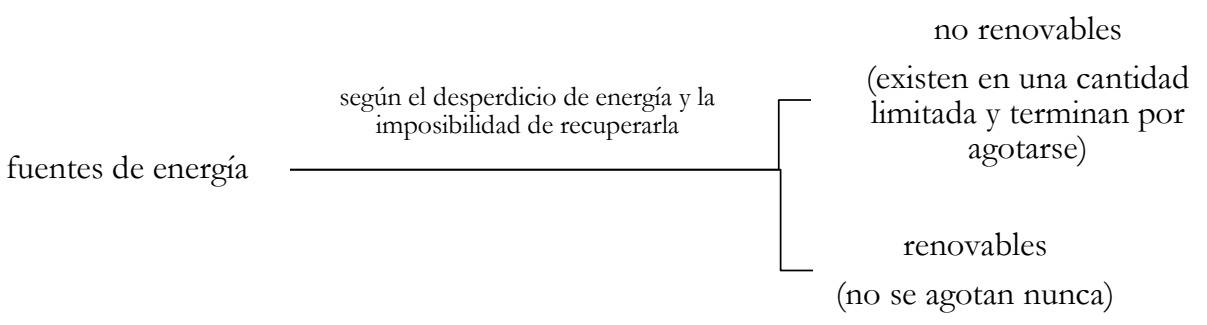

Figura 4. Propuesta de representación gráfica del texto 2.

Tabla 3. Ejemplo de informe composicional.

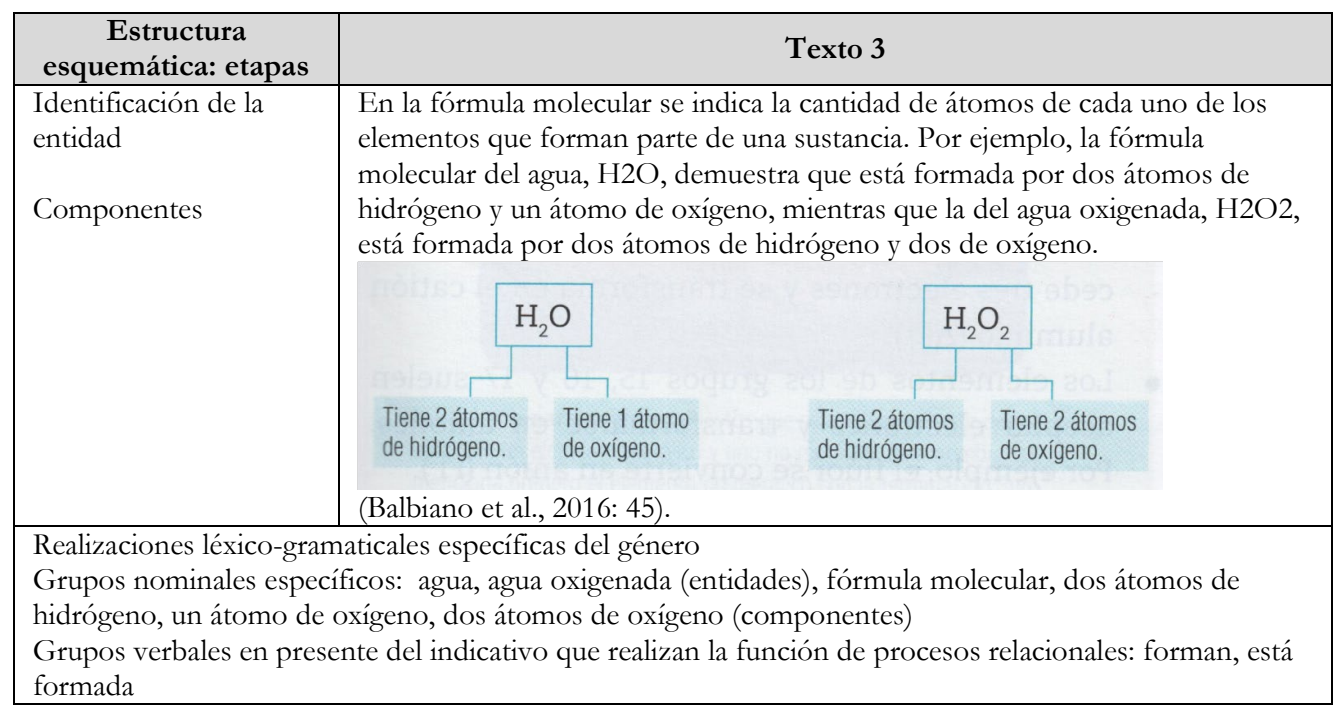




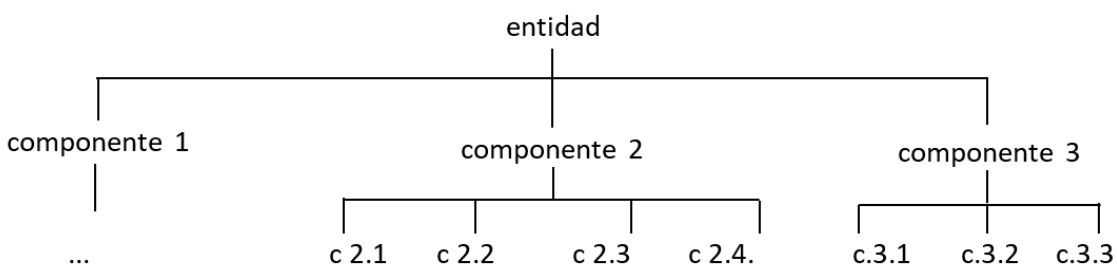

Figura 5. Propuesta de representación gráfica del informe composicional, adaptado de Martin y Rose (2008: 149).

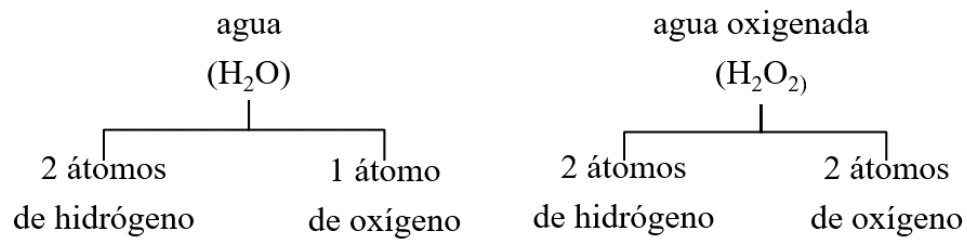

Figura 6. Propuesta de representación gráfica del texto 3.

Tabla 4. Ejemplo de explicación secuencial.

\begin{tabular}{|c|c|}
\hline $\begin{array}{c}\text { Estructura esquemática: } \\
\text { etapas }\end{array}$ & Texto 4 \\
\hline $\begin{array}{l}\text { Secuencia de explicación } \\
\text { (fase 1) }\end{array}$ & $\begin{array}{l}\text { En los reactores nucleares se producen reacciones en cadena de fisión } \\
\text { controlada, en las que los neutrones liberados volverán a producir la fisión } \\
\text { de otros núcleos y así sucesivamente, liberando gran cantidad de energía. } \\
\text { La transformación de la energía nuclear en electricidad ocurre en etapas. } \\
\text { Se produce la fisión de los núcleos de uranio en el reactor nuclear. Esto } \\
\text { emite gran cantidad de energía en forma de calor. } \\
\text { El calor liberado eleva la temperatura del agua en la caldera hasta el punto } \\
\text { de ebullición. } \\
\text { El vapor de agua así formado es conducido hasta unas turbinas que, a su } \\
\text { paso, comienzan a girar. } \\
\text { Un generador transforma la energía cinética en las turbinas en energía } \\
\text { eléctrica, que luego se distribuye para su consumo posterior (Balbiano et } \\
\text { al., 2016, p. } 98 \text { ). }\end{array}$ \\
\hline \multicolumn{2}{|c|}{$\begin{array}{l}\text { Realizaciones léxico-gramaticales específicas del género } \\
\text { Grupos nominales específicos: la transformación de la energía nuclear en electricidad (fenómeno), } \\
\text { reacciones en cadena de fisión controlada, los neutrones liberados, la fisión de otros núcleos, la fisión de } \\
\text { los núcleos de uranio, el calor liberado, el punto de ebullición, el vapor de agua, energía cinética, energía } \\
\text { eléctrica } \\
\text { Procesos nominalizados: transformación, reacciones, fisión, ebullición } \\
\text { Grupos verbales en presente del indicativo que realizan la función de procesos materiales: se producen, } \\
\text { ocurre, emite, eleva, es conducido, transforma } \\
\text { Frases preposicionales: en etapas, en los reactores nucleares, en la caldera, en las turbinas }\end{array}$} \\
\hline
\end{tabular}




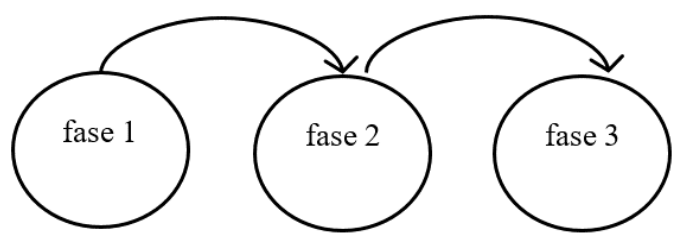

Figura 7. Propuesta de representación gráfica de la explicación secuencial, adaptado de Martin y Rose (2008: 153).

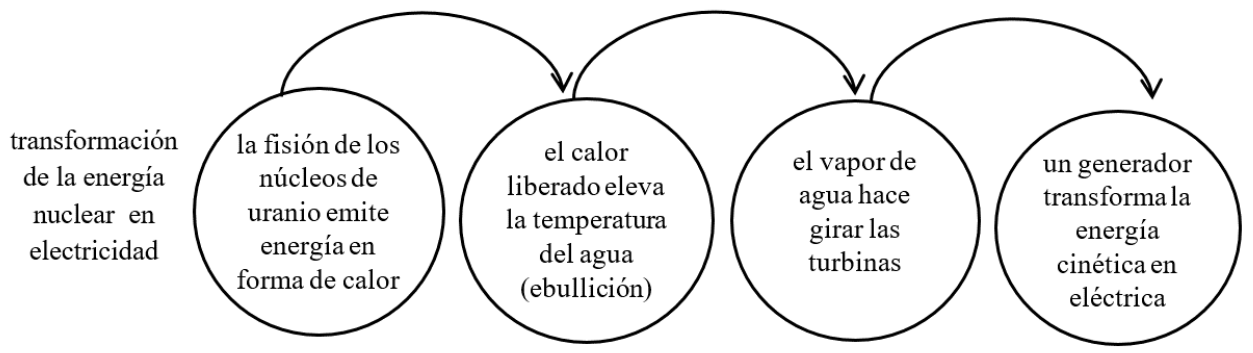

Figura 8. Propuesta de representación gráfica del texto 4.

Tabla 5. Ejemplo de explicación factorial.

\begin{tabular}{|l|l|}
\hline $\begin{array}{c}\text { Estructura esquemática: } \\
\text { etapas }\end{array}$ & \multicolumn{1}{c|}{ Texto 5 } \\
\hline Identificación del fenómeno & $\begin{array}{l}\text { Factores que influyen en la velocidad de las reacciones químicas } \\
\text { Una vez que se produce una reacción cde qué depende que ocurra de } \\
\text { forma más rápida o más lenta? } \\
\text { La velocidad de reacción se define como la cantidad de reactivo que se } \\
\text { consume o de producto que se obtiene en un tiempo determinado. Esta } \\
\text { velocidad depende de varios factores. } \\
\text { Naturaleza de los reactivos. Algunas sustancias reaccionan más rápido que } \\
\text { otras al enfrentarlas al mismo reactivo. Por ejemplo, el zinc reacciona } \\
\text { mucho más rápido que el cobre con el ácido clorhídrico. } \\
\text { Concentración de los reactivos. Cuanto mayor es la concentración de los } \\
\text { reactivos, mayor es la velocidad de una reacción. }\end{array}$ \\
Factor 2 & $\begin{array}{l}\text { Superficie de contacto. Si en lugar de colocar un reactivo sólido lo } \\
\text { dividimos en pequeños trozos o lo pulverizamos, aumenta la superficie de } \\
\text { contacto entre reactivos y la reacción se desencadena más rápido. Por } \\
\text { ejemplo, la lana de acero, formada por hilos finos con alto contenido de } \\
\text { hierro (la conocida virulana), se oxida con gran celeridad porque presenta } \\
\text { una gran superficie en contacto con el oxígeno del aire. } \\
{[\ldots] \text { (Balbiano et al., 2016, p. 63). }}\end{array}$ \\
\hline \multicolumn{2}{|l|}{3} \\
$\begin{array}{l}\text { Realizaciones léxico-gramaticales específicas del género } \\
\text { Grupos nominales específicos: la velocidad de las reacciones químicas (fenómeno), varios factores, } \\
\text { naturaleza de los reactivos, concentración de los reactivos, superficie de contacto (factores) }\end{array}$ \\
$\begin{array}{l}\text { Grupos verbales en presente del indicativo que realizan la función de procesos relacionales: influyen, } \\
\text { depende } \\
\text { Conectores lógicos causales: porque }\end{array}$ \\
\hline
\end{tabular}




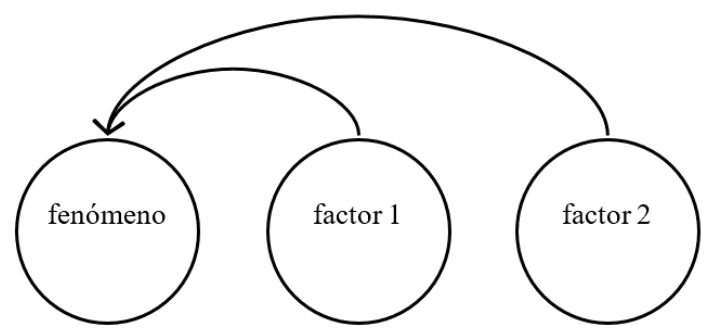

Figura 9. Propuesta de representación gráfica de la explicación factorial, adaptado de Martin y Rose (2008: 159).

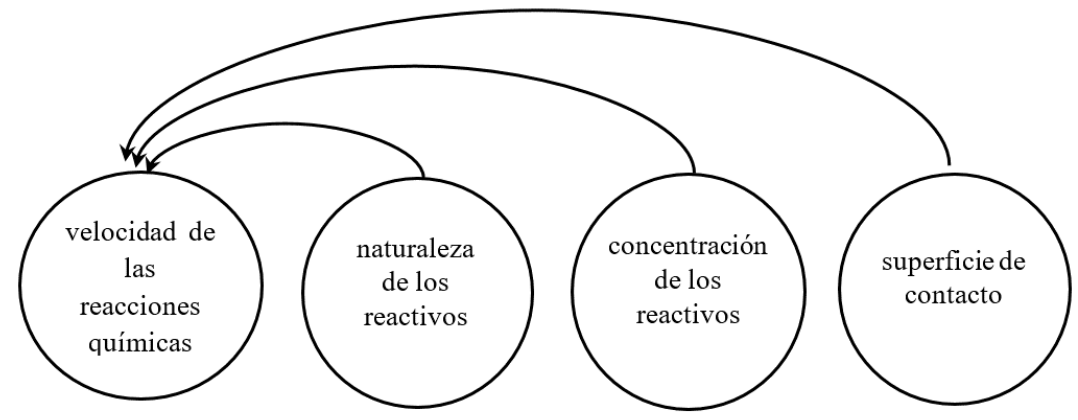

Figura 10. Propuesta de representación gráfica del texto 5 .

Tabla 6. Ejemplo de explicación de consecuencia.

\begin{tabular}{|l|l|}
\hline $\begin{array}{c}\text { Estructura esquemática: } \\
\text { etapas }\end{array}$ & \multicolumn{1}{c|}{ Texto 6} \\
\hline Identificación del fenómeno & $\begin{array}{l}\text { La lluvia ácida es otro fenómeno moderno y es el resultado de gases } \\
\text { emitidos por el hombre que afectan el ambiente e impactan no solo sobre } \\
\text { los ambientes naturales, sino también sobre los urbanos. } \\
{[\ldots]} \\
\text { Algunos de sus efectos son: } \\
\text { acidificación de los cuerpos de agua, que dificulta el desarrollo de la vida } \\
\text { acuática y produce gran mortandad de peces; } \\
\text { afecta directamente a la vegetación y produce graves daños en las zonas } \\
\text { forestales, debido a que los árboles más altos están más expuestos; } \\
\text { corrosión de construcciones, ya que puede disolver el carbonato de calcio }\end{array}$ \\
& $\begin{array}{l}\text { y afectar monumentos y edificaciones construidas con mármol o caliza; } \\
{[\ldots] \text { (Carmona de Rey et al. 2010, p. 41). }}\end{array}$ \\
\hline $\begin{array}{l}\text { Realizaciones léxico-gramaticales específicas del género } \\
\text { Grupos nominales específicos: la lluvia ácida (fenómeno), acidificación de los cuerpos de agua, } \\
\text { mortandad de peces, graves daños en las zonas forestales, corrosión de construcciones (consecuencias) }\end{array}$ \\
$\begin{array}{l}\text { Procesos nominalizados: acidificación, mortandad, corrosión } \\
\text { Grupos verbales en presente del indicativo que realizan la función de procesos materiales: afectan, } \\
\text { impactan, produce }\end{array}$ \\
\hline
\end{tabular}




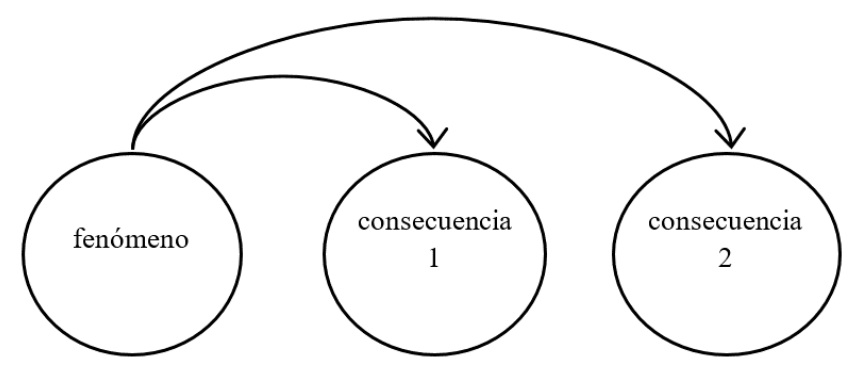

Figura 11. Propuesta de representación gráfica de la explicación de consecuencia, adaptado de Martin y Rose (2008: 160).

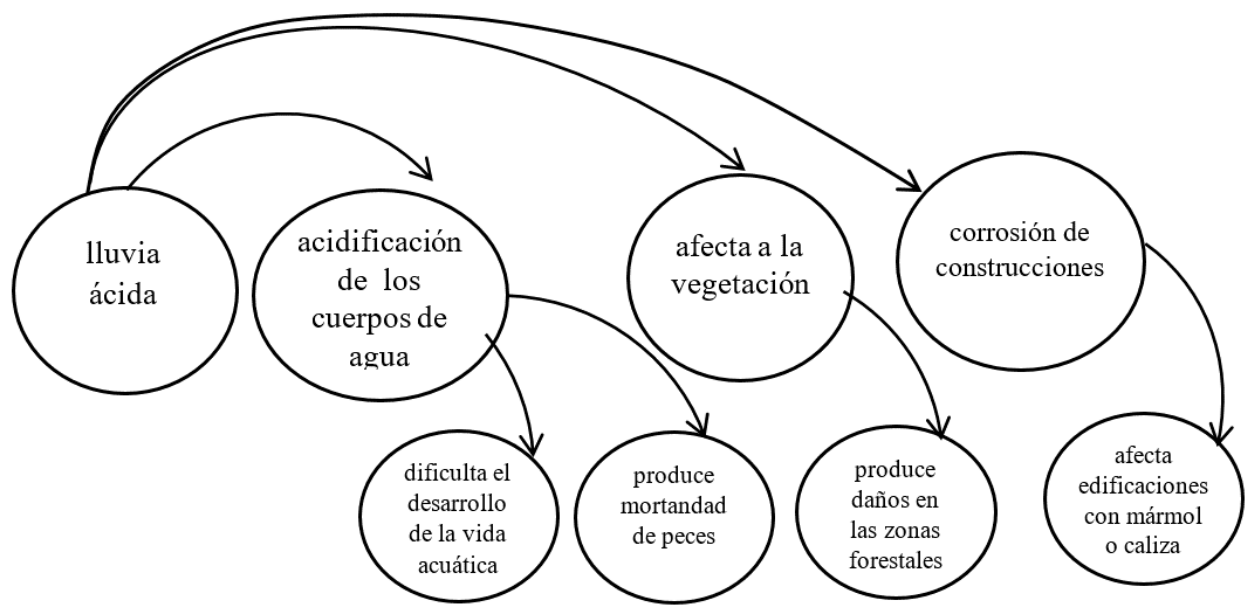

Figura 12. Propuesta de representación gráfica del texto 6. 
Tabla 7. Ejemplo de explicación condicional.

\begin{tabular}{|l|l|}
\hline $\begin{array}{c}\text { Estructura } \\
\text { esquemática: } \\
\text { etapas }\end{array}$ & \multicolumn{1}{c|}{ Texto 7} \\
\hline $\begin{array}{l}\text { Identificación del } \\
\text { fenómeno }\end{array}$ & $\begin{array}{l}\text { Evolución isocórica o a volumen constante. Si el volumen del gas permanece } \\
\text { constante, no hay intercambio de energía por trabajo. Si, en estas condiciones, el gas } \\
\text { absorbe energía por calor, toda la energía transferida hace aumentar la energía } \\
\text { interna del gas, y este aumenta su temperatura. Las moléculas tienen, en promedio, } \\
\text { más energía cinética y, por lo tanto, golpean con más fuerza y con mayor frecuencia } \\
\text { las paredes del recipiente, de modo que la presión aumenta. Esta situación se expresa } \\
\text { de la siguiente manera: } \\
\Delta \mathrm{U}=\mathrm{Q}+\mathrm{L}\end{array}$ \\
$\mathrm{L}=0 \rightarrow \Delta \mathrm{U}=\mathrm{Q}$
\end{tabular}

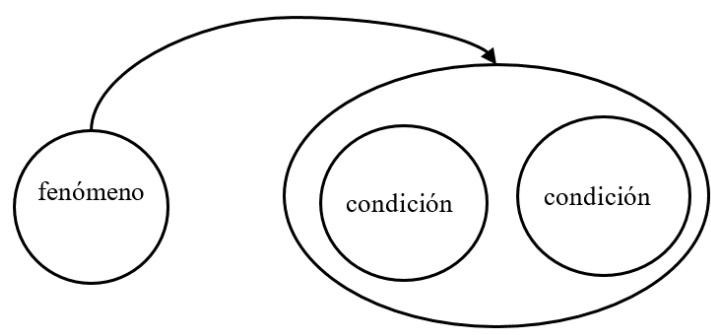

Figura 13. Propuesta de representación gráfica de la explicación condicional, adaptado de Martin y Rose (2008: 157). 


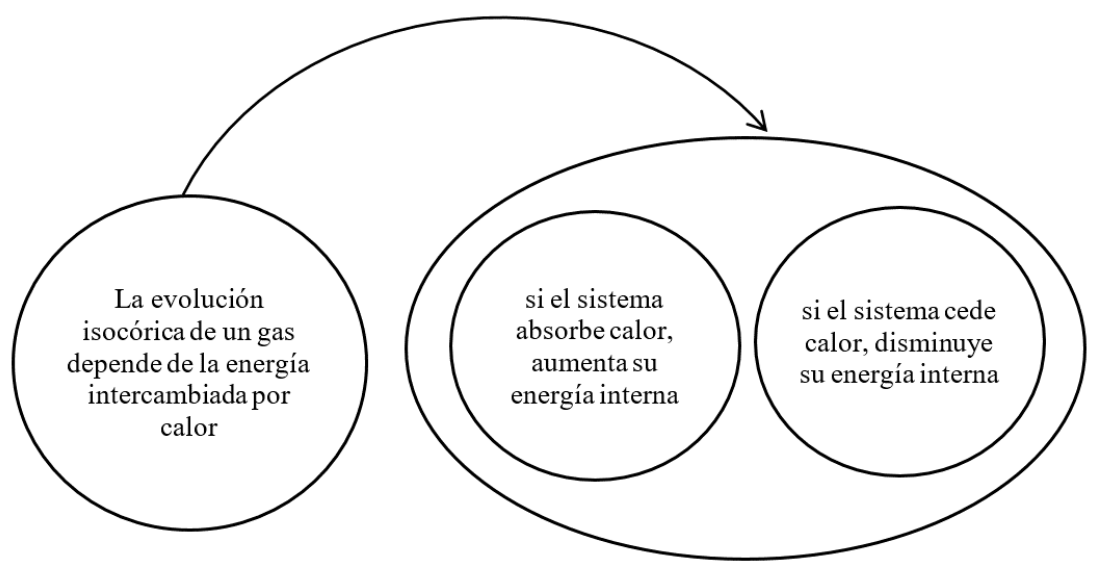

Figura 14. Propuesta de representación gráfica del texto 7.

El análisis realizado de los textos considerados representativos de cada género, evaluado a la luz del objetivo de esta investigación, nos permite afirmar que:

- El informe descriptivo (Tabla 1) se centra en la entidad "plásticos", la cual se destaca con una tipografía diferente del resto del texto, definiendo, ejemplificando y caracterizando este grupo de materiales. Así, las actividades de lectura propicias para los informes descriptivos deberían centrarse en: la identificación del fenómeno y del propósito del texto (¿a qué fenómeno hace referencia el texto?, ¿lo describe, lo clasifica o indica cómo está compuesto?), la determinación de la estructura esquemática y de la representación gráfica que la refleje (¿qué etapas se pueden identificar en la descripción del fenómeno?, ¿qué representación gráfica representa la organización de la información?), la identificación de las realizaciones léxico-gramaticales que caracterizan el género (¿cuáles son los grupos nominales, verbales y adjetivos específicos del género?).

- El informe clasificatorio (Tabla 2) identifica la clase general "fuentes de energía" definiéndola, luego presenta el criterio de clasificación y las subclases, acompañada cada una de ejemplos. En relación con el criterio de clasificación, el mismo contempla dos aspectos "el desperdicio de esta energía" y "la imposibilidad de poder volver a recuperarla". La aplicación del primer criterio es explícita para la subclase "fuentes renovables" ("presentan la ventaja de ser menos contaminante") pero debe ser inferida para la subclase "fuentes no renovables". Cabe destacar que no se mantiene la misma regla al destacar las palabras en negrita cuando se definen las subclases. En este caso, las actividades de lectura para los informes clasificatorios tendrían que centrarse en: la identificación del fenómeno (clase general) y del propósito del texto (¿a qué fenómeno hace referencia el texto?, ¿lo describe, lo clasifica o indica cómo está compuesto?), la determinación de la estructura esquemática y de la representación gráfica que la refleje (¿cuál es el criterio de clasificación?, ¿cuáles 
son las subclases?, ¿qué información se proporciona sobre las subclases?, ¿se definen y/o se ejemplifican?, ¿qué representación gráfica representa la organización de la información?), la identificación de las realizaciones léxicogramaticales que caracterizan el género (¿cuáles son los grupos nominales, verbales y frases preposicionales específicos del género?).

- El informe composicional (Tabla 3) identifica la entidad "la fórmula molecular", la cual se destaca en el texto con letra negrita, y los componentes para los ejemplos del agua y del agua oxigenada. Las imágenes que acompañan el texto muestran la composición de cada una de las fórmulas moleculares, expresada a través de símbolos químicos y subíndices que indican la cantidad de átomos en cada molécula. Las actividades de lectura para los informes composicionales tendrían que incluir la identificación de la entidad (o entidades) y del propósito del texto (¿a qué entidad hace referencia el texto?, ¿la describe, la clasifica o indica cómo está compuesta?), la determinación de la estructura esquemática y de la representación gráfica que la refleje (¿cuáles son los componentes de la entidad?, ¿qué representación gráfica representa la organización de la información?), la identificación de las realizaciones léxicogramaticales que caracterizan el género (¿cuáles son los grupos nominales y verbales específicos del género?).

- La explicación secuencial (Tabla 4) se focaliza en el fenómeno "la transformación de la energía nuclear en electricidad" presentando mediante una enumeración cada una de las fases que constituyen la secuencia, entre las cuales se da una relación causal obligatoria de tal modo que no puede ocurrir una etapa sin que se produzca la anterior. Las relaciones causales entre los eventos que conforman el proceso en este texto son: "la fisión de los núcleos" provoca la emisión de "calor" en la etapa 1; "el calor" lleva el agua al "punto de ebullición" en la etapa 2; "el vapor de agua" que es liberado por el agua en ebullición hace "girar" las turbinas en la etapa 3; y el giro se asocia a la "energía cinética" que se transforma en "energía eléctrica" en la etapa final. En relación con las actividades de lectura para las explicaciones secuenciales, las mismas deberían ayudar a la identificación del fenómeno y del propósito del texto (¿a qué fenómeno hace referencia el texto?, ¿el fenómeno se explica por medio de: una secuencia de eventos, múltiples factores, consecuencias o condiciones?), la determinación de la estructura esquemática y de la representación gráfica que la refleje (¿la secuencia es simple o compleja?; si es simple, ¿hay una relación causal obligatoria entre los eventos?, ¿cuáles son las fases de la explicación?, ¿cada fase es antecedente de la que le sigue?, ¿qué representación gráfica representa la organización de la información?), la identificación de las realizaciones léxico-gramaticales que caracterizan el género (¿cuáles son los grupos nominales, verbales, frases preposicionales y conectores lógicos temporales específicos del género?, ¿hay procesos nominalizados?, ¿qué 
información implícita en el proceso nominalizado hace falta que sea recuperada?).

- La explicación factorial (Tabla 5) explica "la velocidad de las reacciones químicas" en virtud de los factores contribuyentes, destacando todos estos elementos con letra negrita. Comienza definiendo el concepto y, luego, presenta uno a uno cada factor de los cuales, por razones de extensión, solo incluimos los tres primeros. Para cada factor, se analiza la dependencia y, en algunos casos, se incluyen ejemplos. Las actividades de lectura para las explicaciones factoriales deberían apuntar a la identificación del fenómeno y del propósito del texto (¿a qué fenómeno hace referencia el texto?, ¿el fenómeno se explica por medio de: una secuencia de eventos, múltiples factores, consecuencias o condiciones?), la determinación de la estructura esquemática y de la representación gráfica que la refleje (¿qué etapas se distinguen en la organización de la información?, ¿cuáles son los factores contribuyentes?, ¿qué representación gráfica representa la organización de la información?), la identificación de las realizaciones léxico-gramaticales que caracterizan el género (¿cuáles son los grupos nominales, verbales y conectores lógicos causales específicos del género?)

- La explicación de consecuencia (Tabla 6) identifica el fenómeno "la lluvia ácida" definiéndolo en función de sus causas y consecuencias. Posteriormente, el texto se centra en varios de sus efectos los cuales a su vez tienen consecuencias en la naturaleza. El uso de viñetas ayuda a distinguir cada una de las consecuencias del fenómeno. En este caso, las actividades de lectura para las explicaciones de consecuencia tendrían que enfocarse en la identificación del fenómeno y del propósito del texto (¿a qué fenómeno hace referencia el texto?, ¿el fenómeno se explica por medio de: una secuencia de eventos, múltiples factores, consecuencias o condiciones?), la determinación de la estructura esquemática y de la representación gráfica que la refleje (¿qué etapas se distinguen en la organización de la información?, ¿cuáles son las consecuencias del fenómeno?, ¿qué representación gráfica representa la organización de la información?), la identificación de las realizaciones léxico-gramaticales que caracterizan el género (¿cuáles son los grupos nominales y verbales específicos del género?, ¿hay procesos nominalizados?, ¿qué información implícita en el proceso nominalizado hace falta que sea recuperada?).

- La explicación condicional (Tabla 7) explica el fenómeno de la "evolución isocórica" de un gas. Para esto, se identifica en primer lugar el fenómeno asociándolo a una condición fija (que el volumen del gas se mantiene constante) $y$, posteriormente, se indican dos efectos posibles que dependen de dos condiciones variable, es decir, si el gas absorbe energía o si el gas cede energía en forma de calor. Los enunciados del texto son avalados por las ecuaciones que lo explican. Las actividades de lectura para las explicaciones condicionales 
se podrían centrar en la identificación del fenómeno, y del propósito del texto (¿a qué fenómeno hace referencia el texto?, ¿el fenómeno se explica por medio de: una secuencia de eventos, múltiples factores, consecuencias o condiciones?), la determinación de la estructura esquemática y de la representación gráfica que la refleje (¿qué etapas se distinguen en la organización de la información?, ¿cuáles son las condiciones y sus efectos?, ¿qué representación gráfica representa la organización de la información?), la identificación de las realizaciones léxico-gramaticales que caracterizan el género (¿cuáles son los grupos nominales, verbales y cláusulas condicionales específicos del género?, ¿hay procesos nominalizados?, ¿qué información implícita en el proceso nominalizado hace falta que sea recuperada?).

Para cada uno de los siete textos analizados propusimos una representación gráfica de las ideas más relevantes y sus relaciones, teniendo en cuenta -cuando se lo indica en el epígrafe correspondiente de las figuras- la propuesta de Martin y Rose (2008) e introduciendo algunas modificaciones que consideramos que ayudarían a docentes y a estudiantes a poner en evidencia la estructura esquemática de cada género. Resumiendo las principales características de las representaciones gráficas podemos expresar que:

- En el informe descriptivo (Figuras 1 y 2) la entidad que se describe ocupa el lugar de mayor relevancia a la izquierda de la representación; proponemos que la misma se acompañe en el esquema de una idea breve que exprese su definición cuando el texto la incluya. Los rasgos o características de la entidad se desprenden de la misma utilizando líneas contínuas para cada uno. Los ejemplos podrían o no incluirse en la representación por tratarse de información que no necesariamente forma parte del informe descriptivo.

- En el informe clasificatorio (Figura 3) la clase general ocupa el lugar central a la izquierda, derivándose de allí una línea horizontal que se subdivide en tantas otras como subclases existan. La representación se lee de izquierda a derecha, tal como proponen Martin y Rose (2008) en el marco de la LSF. Consideramos conveniente incluir en la representación el criterio de clasificación sobre la línea horizontal y la aplicación del mismo para caracterizar cada subclase (Figura 4). Las subclases se ubican en un mismo nivel vertical.

- En el informe composicional (Figuras 5 y 6) la entidad ocupa el lugar central superior, derivándose de ella una línea vertical que se subdivide en tantas otras como componentes tenga la entidad. La representación está organizada siguiendo una jerarquía arriba-abajo como proponen Martin y Rose (2008). Así, los componentes se ubican sobre un mismo nivel horizontal. En algunos casos, puede ser necesario plantear la composición de algún componente de la entidad. 
Para representar gráficamente las explicaciones, se hace necesario distinguir entre una estructura orbital -con una especie de núcleo del cual dependen otros segmentosy una estructura serial -donde los segmentos dependen unos de otros pero no hay un elemento nuclear- (Martin \& Rose, 2008). En el primer caso, la estructura se considera mononuclear mientras que en el segundo caso es multinuclear. Las principales características de las representaciones gráficas propuestas en este trabajo son:

- En la explicación secuencial (Figura 7) cada una de las fases de la secuencia de explicación se coloca en orden de ocurrencia de derecha a izquierda, y se unen mediante arcos relacionales orientados que apuntan desde una etapa a la que le sigue conformando una estructura serial multinuclear. Proponemos que el fenómeno ocupe un lugar destacado a la izquierda de la representación (Figura 8), y que se escriba cada fase en un círculo intentando resumir el evento más importante ocurrido en dicha etapa del proceso.

- En la explicación factorial (Figuras 9 y 10) el fenómeno ocupa el primer círculo o núcleo de la estructura (estructura orbital mononuclear), donde confluyen los arcos relacionales que lo unen con cada uno de los factores contribuyentes. En este caso, los factores aparecen nominalizados, tal como se presentan en el texto fuente.

- En la explicación de consecuencia (Figuras 11 y 12) el fenómeno ocupa el primer círculo, partiendo desde allí todos los arcos relacionales que lo unen con cada una de las consecuencias que se mencionan en el texto, orientando las flechas en todos los casos desde las causas hacia las consecuencias. Esta estructura también se considera orbital mononuclear. En este caso, como la estructura interna de cada consecuencia presentada en el texto incluye, a su vez, otras consecuencias, la representación gráfica anida otras relaciones (también de consecuencia). En cada círculo se presenta, ya sea en forma de grupo nominal o de grupo verbal las consecuencias del evento, teniendo en cuenta el modo en que se proponen en el texto fuente.

- En la explicación condicional (Figuras 13 y 14) el fenómeno ocupa el primer círculo (estructura orbital), partiendo desde allí un arco relacional que lo une con una elipse mayor que incluye las posibles consecuencias bajo todas y cada una de las condiciones que son consideradas en el texto fuente.

\section{CONCLUSIONES}

Nuestro estudio, que focaliza en la caracterización genérica de los informes y las explicaciones en manuales escolares de ciencias naturales de la educación secundaria argentina para abordar temas de física y de química, permite confirmar que los informes y las explicaciones se utilizan en los textos escolares para presentar los contenidos científicos. Esto se hace en ocasiones en forma pura, lo que ha hecho posible construir la muestra de géneros prototípicos que hemos analizado aquí. 
En lo que se refiere a las categorías de análisis seleccionadas y consideradas en el marco de la LSF, se convierten en potentes lentes para desentrañar la información de los textos escolares. Las ventajas de la herramienta propuesta como resultado de esta investigación se relacionan con que:

- se torna potencialmente adecuada para analizar textos de física y de química de los manuales escolares de la educación secundaria,

- propone al docente de ciencias un acercamiento básico desde el punto de vista de la Lingüística que, sumado a su conocimiento disciplinar, se conjugan para sacar a la luz las relaciones entre las ideas del texto que deberían abordarse y profundizarse en las consignas de las actividades de lectura que planifique para las clases,

- facilita la identificación de las relaciones entre las ideas del texto,

- se convierte en un instrumento para la detección de posibles dificultades del texto para hacer una selección crítica del mismo como recurso didáctico y para jerarquizar la información más relevante del mismo.

Uno de los aportes de la propuesta que se presenta en este artículo se relaciona con la utilidad de la representación gráfica propia de cada uno de los géneros que se abordan. Por una parte, resulta útil para los investigadores, en la medida en que permite que cada uno de los géneros analizados pueda ser esquematizado con un grado de generalización tal que se pueda adaptar para otro texto con características similares en la organización de la información. Por otra parte, se constituye en una herramienta didáctica para el docente de ciencias, ya que al hacer explícita la organización de la información del texto, guiado tanto por los rasgos lingüísticos que logre detectar cómo por su conocimiento del contenido disciplinar, podrá así evaluar la adecuación del texto en lo referido a su suficiencia informativa y detectar si hay dificultades u obstáculos que deban abordarse en la lectura y, en consecuencia, en la enseñanza del contenido. Por último, la construcción de una representación gráfica del texto es conveniente para el estudiante ya que tiene una doble función: requiere analizar en detalle las ideas del texto y sus vinculaciones, y promueve la comprensión del contenido disciplinar al exigir establecer las relaciones entre las ideas expresadas en el mismo.

Una limitación del análisis realizado, que se constituye en uno de los aspectos a estudiar en próximas investigaciones, se relaciona con la inclusión de macrogéneros, es decir, la coexistencia de más de un género en un texto, para abordar muchos de los contenidos escolares de ciencias. Esta complejidad puede relacionarse con la naturaleza de la ciencia misma, en cuanto implica al mismo tiempo observar, describir, comparar, clasificar, analizar, discutir, hipotetizar, teorizar, cuestionar, desafiar, argumentar, diseñar experimentos, seguir procedimientos, juzgar, evaluar, decidir, concluir, generalizar, informar, escribir, comunicar y enseñar en y a través del lenguaje 
de la ciencia (Lemke, 1990). Aun cuando los macrogéneros en los textos de los manuales escolares podrían constituir una dificultad y, al mismo tiempo, un desafío para el docente a la hora de analizar o deconstruir el texto, consideramos que el análisis realizado en este estudio constituiría el paso previo necesario para captar esta complejidad.

A modo de conclusión, podemos afirmar que la propuesta presentada aquí se constituye en un punto de partida para sacar a la luz las relaciones entre las ideas de los textos escolares de ciencias y para diseñar actividades de lectura que ayuden a desentrañarlas. El uso de esta herramienta por parte del docente para planificar el acompañamiento durante la lectura permitiría al estudiante una construcción doblemente provechosa. Por un lado, favorecería un acercamiento específico a géneros utilizados en los textos de ciencias mediante la construcción de conocimiento discursivo y, por otra parte, contribuiría en la detección de las relaciones entre los conceptos y leyes científicas que están involucradas en el aprendizaje disciplinar.

\section{REFERENCIAS BIBLIOGRÁFICAS}

Balbiano, A., Deprati, A. M., Díaz, F. G., Franco, R., Iglesias, M. C. \& Molinari Leto, N. (2016). Física y química 3. La materia: Su estructura y sus transformaciones: Los intercambios de energía. Serie Santillana en línea. Buenos Aires: Santillana.

Bazo, R., Abellán, K. M., Caro, G. \& Sellés, J. (2012). Ciencias naturales 1. Serie Mundo dos punto cero. Buenos Aires: Editorial Tinta Fresca.

Carmona de Rey, C., Pusterla, V. \& Viscarra, I. (2010). Ciencias naturales 1. Colección Logonautas. Buenos Aires: Puerto de Palos.

Christie, F. \& Martin, J. R. (Eds.) (2000). Genres and Institutions: Social Practices in the Workplace \& School. Londres: Continuum.

Derewianka, B. (2013). A new grammar companion for teachers. Sydney: PEETA.

Ghio, E. \& Fernández, M. D. (2008). Lingüistica Sistémico Funcional. Aplicaciones a la lengua española. Santa Fe: Universidad Nacional del Litoral, Waldhuter Editores.

Guirado, A. M., Soliveres, M. A. \& Maturano, C. (2015). El taller como modalidad de trabajo en la formación de docentes de ciencias naturales como alfabetizadores. Actas del Congreso Nacional Cátedra UNESCO para el mejoramiento de la calidad y equidad de la educación en América Latina, con base en la lectura y la escritura. Repositorio Universidad Nacional de Rosario [en línea]. Disponible en: http://rephip.unr.edu.ar/xmlui/handle/2133/4833 
Halliday, M. A. K. \& Matthiessen, C. M. (2004). Halliday's introduction to functional gramar (4th ed.). Londres \& Nueva York: Routledge.

Halliday, M. A. K. \& Martin, J. R. (2005). Writing science: Literacy and discursive power. Londres: Taylor \& Francis.

Ibáñez, R., Moncada, F. \& Arriaza, V. (2018). Recontextualización del conocimiento en textos escolares chilenos. Revista Signos. Estudios de Lingüistica, 51(98), 430456.

Ibañez, R., Moncada, F., Cornejo, F. \& Arriaza, V. (2017). Los géneros del conocimiento en textos escolares de educación primaria. Calidoscópio, 15(3), 462-476.

Lemke, J. L. (1990). Talking science: Language, learning, and values. Norwood: Ablex Publishing.

Lerner, D., Aisenberg, B. \& Espinoza, A. (2010). La lectura y la escritura en la enseñanza de ciencias naturales y de ciencias sociales. Una investigación en didácticas específicas. En J. Castorina \& V. Orce (Coords.), Anuario del Instituto de Investigaciones en Ciencias de la Educación. Buenos Aires: FFyL. UBA.

Manghi, D. (2013). La mediación del profesor especialista para la alfabetización semiótica en el aula de matemática. En E. Moyano (Coord.), Aprender ciencias y bumanidades: Una cuestión de lectura y escritura. Aportes para la construcción de un programa de inclusión social a través de la educación lingüistica (pp.199-229). Los Polvorines: UNGS.

Manghi, D., Torres, D. G., Urrutia, E. E., Martínez, C. M., Vega, P. R. \& Morales, V. G. (2016). Leer para aprender a partir de textos multimodales: Los materiales escolares como mediadores semióticos. REXE-Revista de Estudios y Experiencias en Educación, 12(24), 77-91.

Martin, J. R. \& Rose, D. (2008). Genre relations. Mapping culture. Londres: Equinox.

Maturano, C. (2018). El manual escolar en la enseñanza y el aprendizaje de las Ciencias Naturales. Tesis Doctoral, Universidad Nacional de Cuyo, Mendoza, Argentina.

Maturano, C. \& Mazzitelli, C. (2018). Libros de texto de ciencias naturales, de ayer, de hoy y ¿de siempre? Revista de Enseñanza de la Física, 30(1), 49-62.

Maturano, C., Rudolph, C. \& Soliveres, M. A. (2016). El texto del manual escolar de ciencias: ¿Puente u obstáculo para el aprendizaje? Revista de Enseñanza de la Física, 28(Número Extra), 29-37. 
Maturano, C., Soliveres, M. A., Guirado, A. M. \& Quiroga, D. (2014). El docente de Ciencias Naturales como alfabetizador de textos de manuales escolares. IV Jornadas Nacionales y II Latinoamericanas de Investigadores/as en Formación en Educación. IICE, Facultad de Filosofía y Letras. UBA. Construcción de conocimientos y saberes (pp. 281-291). Buenos Aires, Argentina. ISBN 978-987-3617-61-4 [en línea]. Disponible en: http://iice.institutos.filo.uba.ar/actas

Ministerio de Educación y Deportes (MED) (2017). Aprender 2016 Informe de resultados. Buenos Aires: Presidencia de la Nación.

Moss, G., Natale, L. \& Oteiza, T. (2009). El Lenguaje de los textos escolares, el aprendizaje, la ideología y la formación ciudadana. Revista DELTA, 25(Especial), 549-753.

Moyano, E. I. (Coord.) (2013). Aprender ciencias y bumanidades: Una cuestión de lectura y escritura. Aportes para la construcción de un programa de inclusión social a través de la educación lingüística. Buenos Aires: Universidad Nacional de General Sarmiento.

Occelli, M. \& Valeiras, N. (2013). Los libros de texto de ciencias como objeto de investigación: Una revisión bibliográfica. Enseñanza de las Ciencias, 31(2), 133 152.

Parodi, G. (2010). Multisemiosis y lingüística de corpus: Artefactos (multi)semióticos en los textos de seis disciplinas en el corpus PUCV-2010. Revista de Lingü̈stica Teórica y Aplicada, 48(2), 33-70.

Rose, D. \& Martin J. R. (2012). Learning to write, reading to learn. Genre, knowledge and pedagogy in the Sydney School. Sheffield: Equinox.

Schleppegrell, M. J. (2008). The language of schooling. A functional linguistics perspective. Londres: Taylor \& Francis e-Library.

Schleppegrell, M. J. \& Colombi, M. C. (2002). Developing advanced literacy in first and second languages: Meaning with power. Nueva Jersey: Lawrence Erlbaum.

Serrano, R. M., Moraga, M. \& Lazo, L. (2011). Análisis taxonómico de los libros de texto para la enseñanza de Química en educación media. Diálogos educativos, 22, 38-68. 
Soliveres, M. A., Maturano, C. \& Quiroga, D. (2015) ¿Qué actividades proponen los docentes de Ciencias Naturales para guiar la lectura de textos disciplinares? Actas del Congreso Nacional Cátedra UNESCO para el mejoramiento de la calidady equidad de la educación en América Latina, con base en la lectura y la escritura, Subsede Universidad de Rosario: La lectura y la escritura en las sociedades del siglo XXI (Repositorio Universidad Nacional de Rosario) [en línea]. Disponible en: http://rephip.unr.edu.ar/xmlui/handle/2133/4854

Taddei, F. P., Sobico, C. \& Righini, R. (2015). Física 4 ES. La energía. Boulogne: Estrada.

Taibu, R., Rudge, D. \& Schuster, D. (2015). Textbook presentations of weight: Conceptual difficulties and language ambiguities. Phys. Rev. ST Phys. Educ. Res., $11(1), 010117$.

Veel, R. (2000). Learning how to mean-scientifically speaking: Apprenticeship into scientific discourse in the secondary school. En F. Christie \& J. R. Martin (Eds.), Genre and institutions: Social processes in the workplace and school (pp. 161195). Londres: Continuum.

Veel, R. (2005). The greening of school science: Ecogenesis in secondary classrooms. En J. R. Martin \& R. Veel (Eds.), Reading Science. Critical and Functional Perspectives on Discourse of Science (pp.115-152). Londres: Taylor \& Francis eLibrary.

Wignell, P., Martin, J. R. \& Eggins, S. (2005). The discourse of Geography: Ordering and explaining the experiential world. En M. A. K. Halliday \& J. R. Martin (Eds.), Writing Science. Literacy and Discursive Power (pp. 151-184). Londres: Taylor \& Francis e-Library. 